Research Article

\title{
Simulation and Computational Study of CFD on Tube MBR Membrane Assembly
}

\author{
Kun Feng, Chunqing Li $\mathbb{D}^{D}$, Ming Zhang, and Xuting Liu \\ School of Computer Science and Technology, Tiangong University, Tianjin 300387, China \\ Correspondence should be addressed to Chunqing Li; franklcq@163.com
}

Received 22 January 2021; Accepted 29 April 2021; Published 13 May 2021

Academic Editor: Zi-Peng Wang

Copyright (c) 2021 Kun Feng et al. This is an open access article distributed under the Creative Commons Attribution License, which permits unrestricted use, distribution, and reproduction in any medium, provided the original work is properly cited.

\begin{abstract}
When using tubular MBR to treat sewage, the water production is an important parameter to measure the efficiency of the tubular MBR system. The problem to be solved in this paper is to calculate the water yield of the tubular MBR system, so as to evaluate the sewage treatment efficiency of the MBR system. This research uses the CFD simulation software ANSYS 16.0 to study the water yield of the tubular MBR system. The MBR model of a single membrane filament tube was established using the ICEM CFD preprocessor in ANSYS 16.0, and the structured grid was divided to obtain a grid file. Then, the fluid solver was used to solve the mesh file and through the flow monitoring window to obtain the water output of the tubular MBR system. Finally, the CFD postprocessor in ANSYS 16.0 was used to visualize the calculation results and compare them with the waste-water treatment results of some actual MBR systems. The results show that the water yield calculated by the fluent solver is basically the same as that of the actual MBR system. This research realizes the purpose of calculating the water yield of the tubular MBR system with CFD technology, solves the problem of evaluating the working efficiency of the tubular MBR system with water consumption, and realizes the MBR before deployment The evaluation of the working efficiency of the system has certain reference value for the planning, design, and deployment of MBR.
\end{abstract}

\section{Introduction}

Membrane bioreactor (MBR) is a combination of membrane separation technology and biological treatment technology. It has the advantages of stable effluent quality, small floor area, and easy maintenance and management. The tubular MBR membrane module is one of the commonly used membrane modules in the MBR system, so this paper analyzes the tubular MBR membrane module. In the tubular MBR membrane module, the deployment of ceramic membrane will affect the working efficiency of the tubular MBR membrane module, and the water yield is an important parameter to measure the working efficiency of the tubular MBR membrane module, so the problem to be solved in this paper is how to calculate the water yield of the tubular MBR membrane module.

In order to calculate the water yield of tubular MBR membrane module, this paper uses the computational fluid dynamics (CFD) simulation software ANSYS 16.0 to model and calculate the tubular MBR membrane module. ANSYS
16.0 is mainly composed of the ICEM CFD preprocessor, FLUENT solver, and CFD-postprocessor. In the research process, firstly, the geometry model of a single membrane fiber tube MBR membrane module is built with ICEM CFD preprocessor, and the mesh of the geometry model is divided into fine meshes to generate mesh files. Then, the grid file is solved by fluent solver. Because the research object of this paper is to calculate the water yield of MBR membrane module, a flow monitoring window is set at the outlet of the model to observe the water yield. Finally, the CFD postprocessor is used to visualize the calculation results. On this basis, in order to verify the correctness and reliability of fluent calculation results, this paper compares the simulation results with the actual MBR processing results.

\section{Tubular MBR Membrane Module}

The basic structure of the tubular MBR membrane module is shown in Figure 1 [1]. The tubular MBR membrane module 
is mainly composed of a ceramic membrane and component housing. There are many small holes on the wall of the ceramic membrane, and these small holes are used to filter out suspended particles in the sewage [2]. When the tubular MBR system is operated, under the action of the water pump, the waste water which has been pretreated flows in from one end of the ceramic membrane and the filtered waste water flows from the small holes of the ceramic membrane wall to the component housing [3]. Finally, the staff can collect the filtered waste water at the outlet of the component housing. The concentrate formed by the solid suspension in the waste water flows out from the other end of the ceramic membrane. Compared with the traditional curtain MBR membrane module, the tubular MBR membrane module has the advantages of simple structure, strong adaptability, convenient cleaning, high pressure resistance, suitable for treating high viscosity, and high solid content liquid [4]. Therefore, the tubular MBR membrane modules are widely used in MBR waste-water treatment, and this paper uses CFD technology to simulate and calculate the tubular MBR membrane module [5].

\section{CFD Simulation Software ANSYS 16.0}

Computational fluid dynamics (CFD) is an interdisciplinary in fluid mechanics, mathematics, and computer science [6]. It can simulate and analyze various problems in practical engineering to solve various practical problems [7]. At present, there are many kinds of software for CFD simulation on the market, and ANSYS 16.0 is one of the commonly used CFD simulation software. ANSYS16.0 is mainly composed of ICEM CFD preprocessor, FLUENT solver, and CFD postprocessor. The main function of the preprocessor is to create geometric models and finely mesh the geometric models [8]. The main function of the solver is to calculate the mesh file, and the solution process is shown in Figure 2. The main function of the postprocessor is to present the calculation result of the solver in a graphical or image manner so that the user can more intuitively understand the CFD calculation result. In this paper, the CFD simulation software ANSYS 16.0 is used to simulate and calculate the tubular MBR membrane module.

\section{Simulation of CFD on Tubular MBR}

4.1. Preprocessor (ICEM CFD) Establishes a Tubular MBR Geometry Model. The tubular MBR geometric model of a single membrane tube established by ICEM CFD preprocessor is shown in Figure 3, and there are many small holes on the wall of the ceramic membrane [9]. Due to the limited effect of the preprocessor opening small holes, this paper sets the porous boundary conditions in the boundary conditions of the solver. During the operation of the model, the pretreated waste water flows into the ceramic membrane from the inlet and the sewage filtered through the ceramic membrane flows from the small holes of the wall surface of the ceramic membrane to the outlet of the component housing. Due to the entrapment of the ceramic tube wall, the concentrate composed of a large molecule particle or the like flows out from the other end of the ceramic film [10]. The following is a structured meshing of the geometric model.

Since the FLUENT solver is solved in units of grids, the geometry model needs to be meshed in the preprocessor. In ICEM CFD, there are two types of meshing: structured meshing and unstructured meshing. Structured meshing usually divides the geometric model into several quadrilaterals or hexahedrons. Unstructured meshing usually divides the geometric model into several triangles or tetrahedrons. In structured meshing, the determinant $2 \times 2 \times 2$ mesh quality map can measure the quality of the meshing. The principle of determinant $2 \times 2 \times 2$ mesh quality is to characterize the deformation of the unit by calculating the Jacobian determinant value of each hexahedron and then normalizing the matrix of the determinant. The value of one indicates an ideal hexahedral cube, and the value of zero indicates an inverse cube with a negative volume. The mesh quality is represented by the $x$-axis and all cells are between 0 and 1 . If the value of a cell determinant is zero, this cube has one or more degenerate edges. In general, the determinant value above 0.3 can be accepted by most solvers. Since the mass distribution of the ceramic membrane is uniform, the paper uses structured mesh division, in which the O-shaped segmentation operation is performed on the cylindrical part of the geometric model. Finally, the resulting mesh file is shown in Figures 4 and 5 and is a determinant $2 \times 2 \times 2$ mesh quality map with a minimum mesh mass of 0.621 . Therefore, the meshing of the geometric model is correct. Then, save the mesh file and prepare to solve the mesh file.

4.2. FLUENT Solver Calculates Mesh File. In this section, the mesh file in Section 4.1 is imported into the solver FLUENT, and then, the solution model description and solution process control operations are, respectively, performed in the FLUENT operation tree [11]. In solving the model description, the first step is to check the mesh file to ensure that the minimum cell volume is not negative and select a pressure-based steady-state solver and set the gravitational acceleration. The second step is selecting the basic physical model. Since the flow rate of the sewage in the tubular MBR system is low and the Reynolds number is lower than 2300, the flow state is set to the laminar flow model. The third step is to introduce fluid material from the material library and use water as the fluid material. The fourth step is setting the calculation area properties and sets the water as the calculation domain working medium. The fifth step is setting the calculation domain boundary conditions. First, the "Mass-Flow Inlet" is set as the inlet boundary condition and enters the water intake and operating pressure of the tubular MBR in "Mass-Flow Inlet" (these two parameters are provided by the sewage treatment plant.). Then, set "Outflow" to the outlet boundary condition. Finally, the "porous jump" is set to the wall boundary condition of the ceramic membrane, that is, many small holes are opened on the wall surface of the ceramic membrane. After these operations are completed, this paper will solve the process control operation.

In the solution process control, first, we set the solution method. In the drop-down list of pressure-velocity coupling 


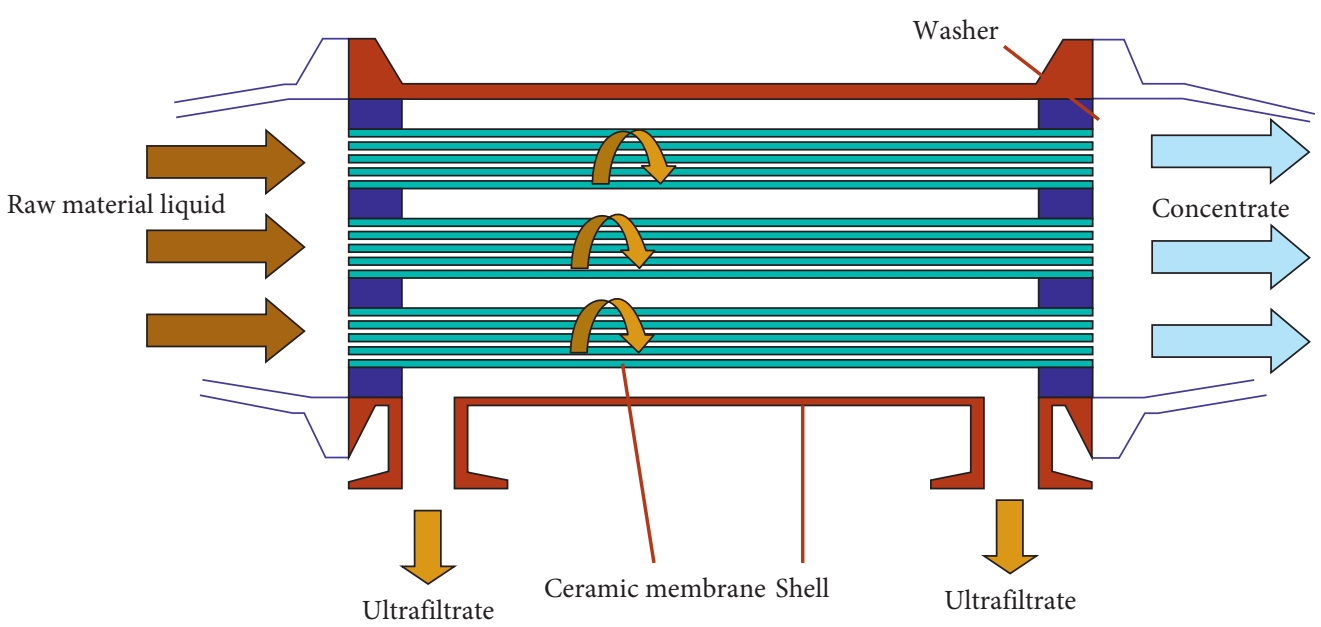

FIgure 1: Tubular MBR structure.

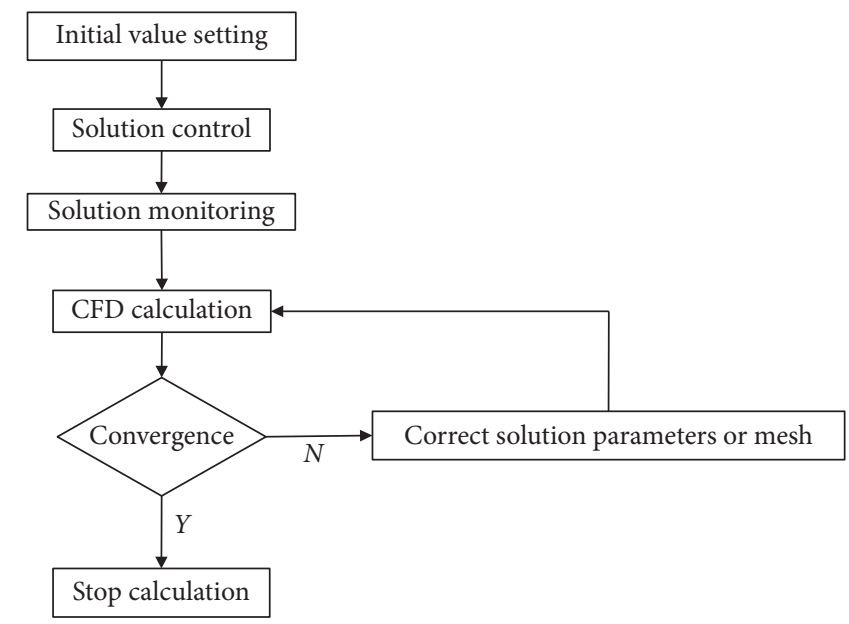

Figure 2: FLUENT solution process block diagram.

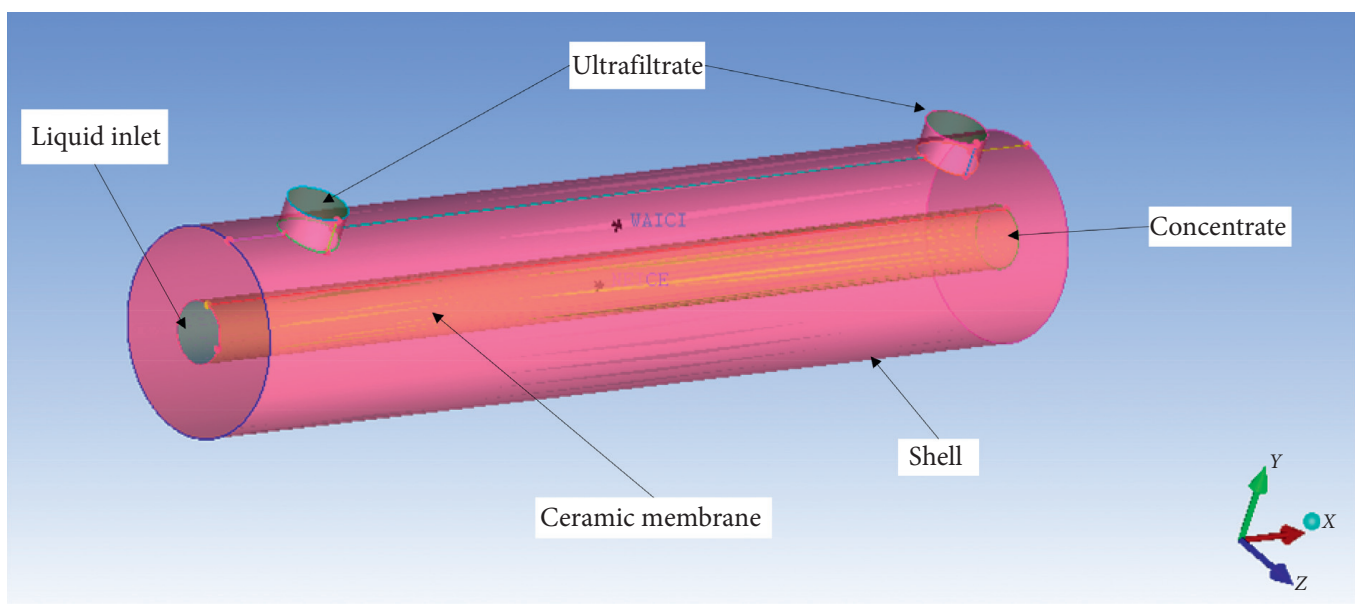

FIgURE 3: Tubular MBR geometry model.

mode, the SIMPLE algorithm is selected to calculate the mesh file; in the microseparation format option, the gradient is set to the unit-based least squares method. The pressure is set to a second-order format and the momentum equation is set to a second-order upwind style. Secondly, we set the convergence threshold and the monitoring window. Since the research object of this paper is to calculate the water production of the tubular MBR, then this article sets the 


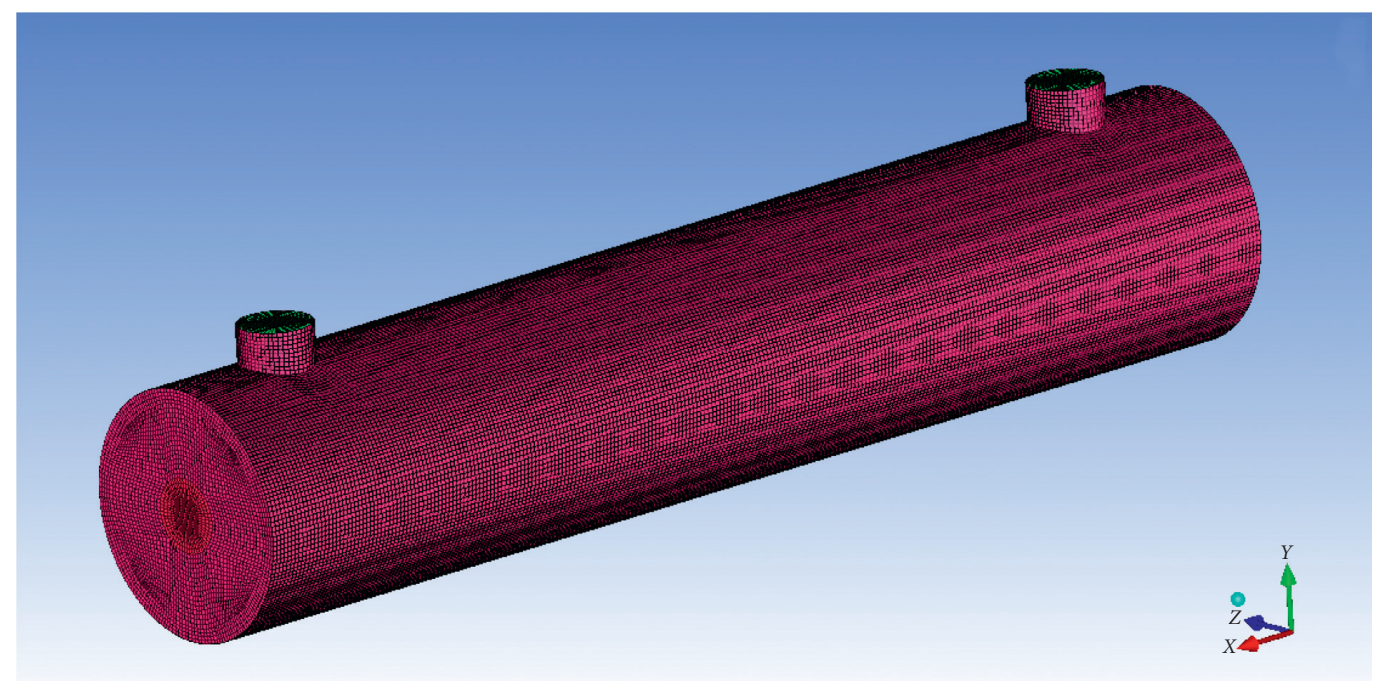

FIGURE 4: The resulting mesh file.

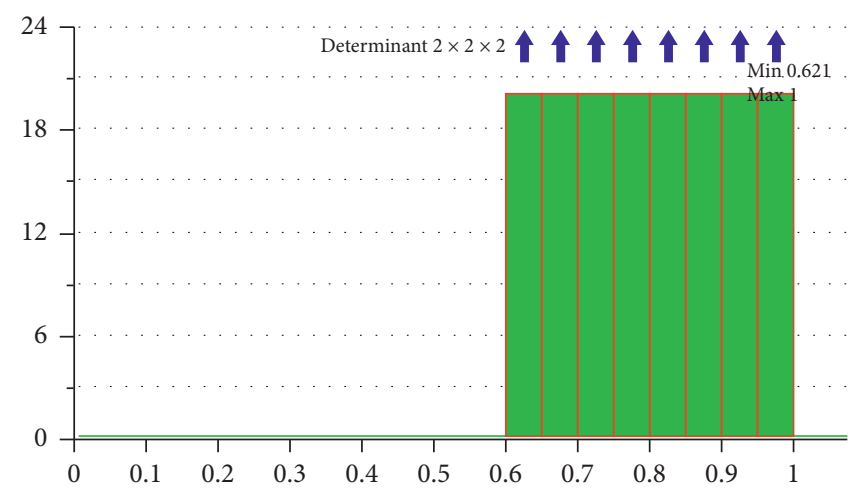

Figure 5: Determinant $2 \times 2 \times 2$ mesh quality map.

traffic monitoring window at the model exit (the mass flow rate is positive for inflow and negative for outflow). And, we set the convergence threshold to 0.001 . Then, initialize the flow field and start the iterative calculation. Figure 6 shows the residual curve in the iterative calculation process of the solver. When the residuals of the velocity in the $x$-axis, $y$ axis, and $z$-axis and the continuity of the continuity equation are not greater than the convergence threshold, the solver's calculation reaches convergence. Figure 7 represents the flow at the outlet of the mesh file during the calculation, that is, the water production of the tubular MBR [12]. Finally, the calculation result is saved, and the postprocessing operation is prepared.

\subsection{Postprocessor (CFD-Post) Visualization Calculation} Results. The postprocessor can present the calculation result in a graphical or image manner. With the postprocessor, the user can effectively observe and analyze the calculation result, thereby more intuitively understanding the calculation result of the solver. Due to the poor performance of the built-in postprocessor in FLUENT, this paper uses CFDPost for postprocessing operations. In CFD-Post, the
FLUENT calculation results are first imported, establishing the flow graph and the pressure cloud map at different sections of the model. Figure 8 is a streamline diagram of water in the model file, where the colored lines on the left represent the magnitude of the velocity, which decreases from top to bottom. And, the flow direction and speed of the water are basically the same as those of the actual tubular MBR system. Figures 9 and 10 are pressure cloud diagrams of the model in which the colored lines on the left side represent the magnitude of the pressure, which decreases sequentially from top to bottom. Observing Figures 9 and 10 which is not difficult to find that since there is a large operating pressure at the entrance of the model, the pressure increases as the process approaches the entrance to the model, which is consistent with the operating principle of the actual tubular MBR. Finally, in order to verify the correctness and reliability of the calculation results, this paper has carried out a lot of experimental analysis.

4.4. Experiment Analysis. In order to verify the correctness and reliability of the FLUENT solution results, this paper selects a large number of actual MBR system operation data 


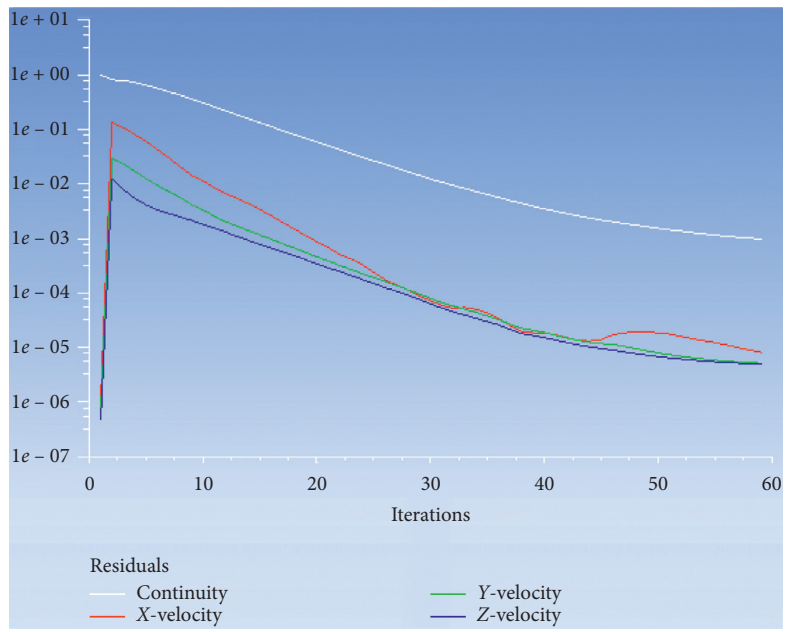

Figure 6: Residual curve.

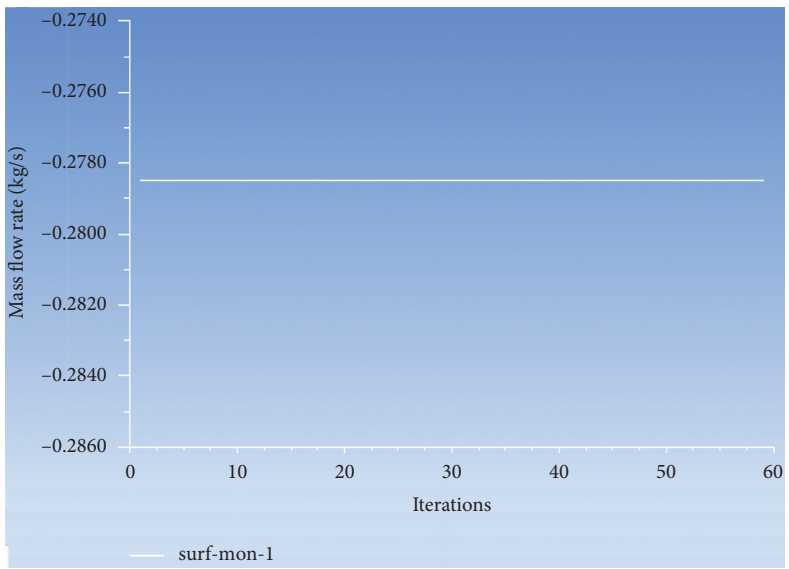

Figure 7: Flow of fluid at the exit of the mesh file.

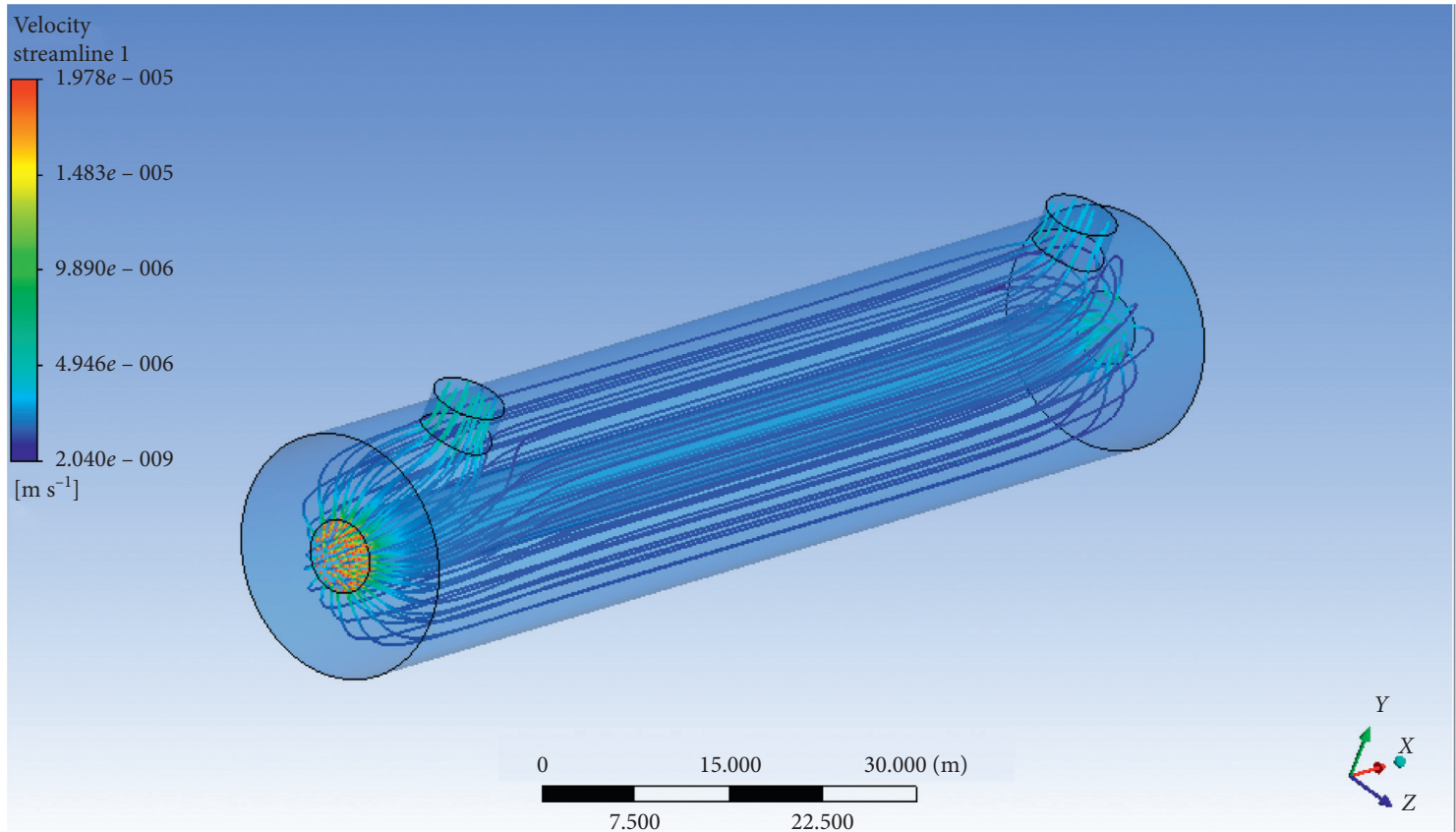

FIgURE 8: Flow diagram of water in the model. 


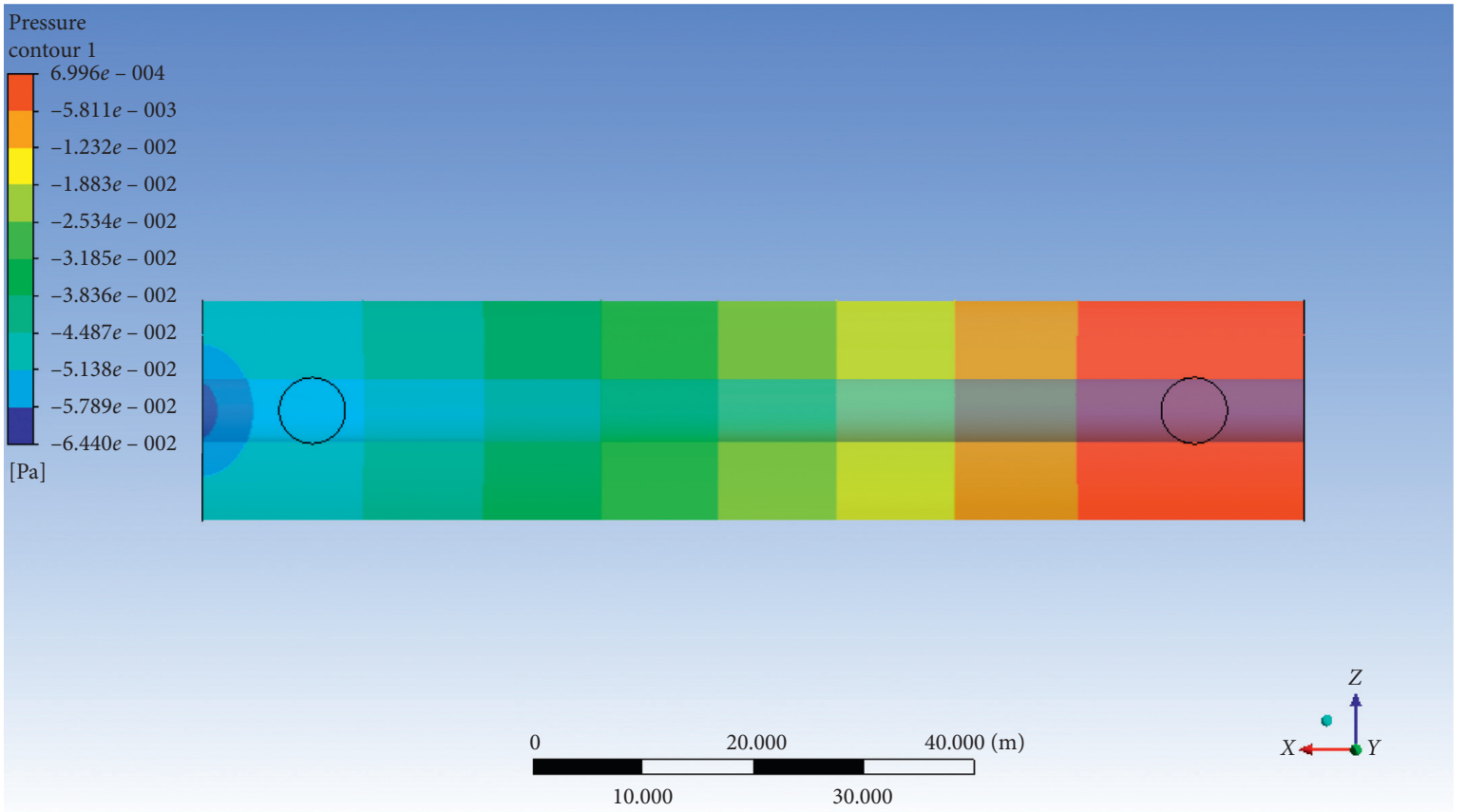

Figure 9: Pressure cloud diagram of the model cross section.

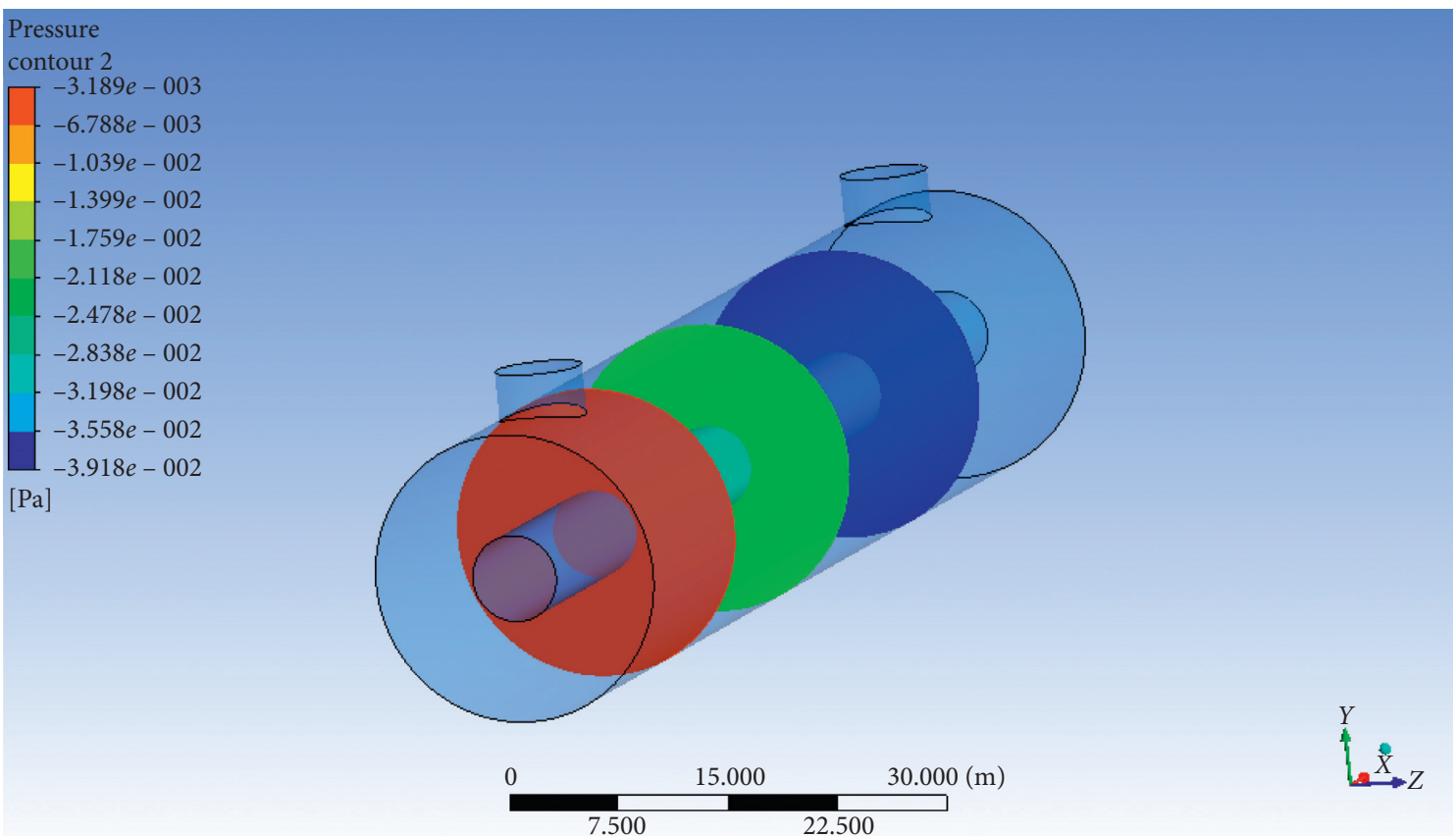

Figure 10: Model different position processing average pressure maps.

of a sewage treatment plant in Shijiazhuang for experimental analysis. In the analysis, the paper divides the actual data, then inputs different water inflows into the inlet boundary conditions of the solver, and starts the iterative calculation. After the calculation, the calculation results obtained by the solver flow monitoring window are compared with the actual tubular MBR system, and the comparison result is shown in Table 1. The comparison results show that the calculation results of the solver are basically the same as those of the actual tubular MBR system, which solves the problem of calculating the water production of tubular MBR model by CFD and achieves the purpose of simulating tubular MBR with CFD, so the calculation result is correct and reliable. 
TABLE 1: Internal pressure series MBR system water production scale.

\begin{tabular}{lccc}
\hline Water intake $\left(\mathrm{m}^{3} / \mathrm{h}\right)$ & TMP $(\mathrm{Kpa})$ & Actual water production $\left(\mathrm{m}^{3} / \mathrm{h}\right)$ & Simulated water production $\left(\mathrm{m}^{3} / \mathrm{h}\right)$ \\
\hline 2 & 17 & 1 & 1.008 \\
1 & 17 & 0.5 & 0.5004 \\
2.5 & 17 & 1.25 & 1.2492 \\
4 & 17 & 2 & 1.99836 \\
3 & 17 & 1.5 & 1.49508 \\
4.5 & 17 & 2.25 & 2.24964 \\
3.5 & 17 & 1.75 & 1.74816 \\
5 & 17 & 2.5 & 2.50488 \\
7 & 17 & 3.5 & 3.4956 \\
9 & 17 & 4.5 & 4.50036 \\
\hline
\end{tabular}

\section{Conclusion}

Because the deployment of membrane will affect the water yield of membrane module of tubular MBR, this paper uses the CFD simulation software ANSYS 16.0 to simulate and calculate the tubular MBR. In the process of analysis, firstly, the tube MBR model of a single membrane wire is constructed by ICEM CFD, and the mesh file is obtained by fine mesh division. Then, the fluent solver is used to solve the grid file, and the water yield is obtained. Finally, CFD-post is used to present the calculation results in a graphical way. On this basis, this paper compares the calculation results of the solver with the operation results of the actual MBR system, and the comparison results show that the calculation results of the solver are basically consistent with the operation results of the actual MBR system, realizing the calculation of the water yield of the tubular MBR membrane module, solving the problem of measuring the working efficiency of the tubular MBR system with the water yield, and realizing the work efficiency of the MBR system before deployment. The evaluation has certain reference value for the planning, design, and deployment of MBR. It has certain value and significance for the research of MBR simulation in the future.

\section{Data Availability}

The data used to support the findings of this study are included within the supplementary information file.

\section{Conflicts of Interest}

The authors declare that they have no conflicts of interest.

\section{Authors' Contributions}

Feng Kun and Zhang Ming substantially contributed to the conception or design of the work and the acquisition, analysis, or interpretation of data for the work. Li Chunqing and Feng Kun drafted the work or revised it critically for important intellectual content. Liu Xuting approved the final version to be published.

\section{Acknowledgments}

This work was supported in part by the National Natural Science Foundation of China (Grant no. 51378350), the National Natural Science Foundation of China (Grant no.
50808130), and the National Natural Science Foundation of China (Grant no. 21506159).

\section{Supplementary Materials}

The supplementary literature is the data of a sewage treatment plant, which is used to set the boundary conditions in fluent to draw the conclusion of the paper. (Supplementary Materials)

\section{References}

[1] T. Vo, J.-J. Lee, J.-S. Kang, S. Park, and K. Han-Seung, "Nitrogen removal by sulfur-based carriers in a membrane bioreactor (MBR).” Membranes, vol. 8, no. 4, 2018.

[2] S. Kitanou, M. Tahri, B Bachiri et al., "Comparative study of membrane bioreactor (MBR) and activated sludge processes in the treatment of Moroccan domestic wastewater," Water Science and Technology, vol. 78, no. 5, 2018.

[3] M. Sabaghian, M. R. Mehrnia, M. Esmaieli, and D. Noormohammadi, "Formation and performance of selfforming dynamic membrane (SFDM) in membrane bioreactor (MBR) for treating low-strength wastewater," Water Science and Technology, vol. 78, no. 4, 2018.

[4] T. Miura, S. Julien, Le Saux Jean-Claude, M. Le, and S. Le Guyader Françoise, "Virus type-specific removal in a full-scale membrane bioreactor treatment process," Food And Environmental Virology, 2017.

[5] F. Yang, Y. Wang, L. Gillerman et al., "Analysis of membrane bioreactor performance for wastewater treatment using ranking methods," Toxicological \& Environmental Chemistry, vol. 99, no. 7-8, 2017.

[6] D. Gregor, "Wehinger. Radiation matters in fixed-bed CFD simulations," Chemie Ingenieur Technik, vol. 91, no. 5, 2019.

[7] T. Raja, "Coupled CFD-FE analysis for the exhaust manifold to reduce stress of a direct injection-diesel engine," International Journal of Ambient Energy, vol. 40, no. 4, 2019.

[8] S. Chapela, J. Porteiro, M. Garabatos, D. Patiño, M. A. Gómez, and J. L. Míguez, "CFD study of fouling phenomena in smallscale biomass boilers: experimental validation with two different boilers," Renewable Energy, p. 140, 2019.

[9] Z. Miao, S. Kuang, H. Zughbi, and A. Yu, "CFD simulation of dilute-phase pneumatic conveying of powders," Powder Technology, p. 349, 2019.

[10] S. Agahzamin and L. Pakzad, "CFD investigation of the gas dispersion and liquid mixing in bubble columns with dense vertical internals," Chemical Engineering Science, 2019. 
[11] M. Behrang, M. Shirvani, and S. H. Hashemabadi, "Multihelical-channel dust separator: CFD simulation and experiment," Chemical Engineering Research and Design, 2019.

[12] Xi Chu, W. Chen, Y. Shang, J. Hao, and De Zhang, "CFD investigation on reverse flow characteristics in U-tubes under two-phase natural circulation," Progress in Nuclear Energy, p. 114, 2019. 